\title{
Learning styles comparison based on a classification methodology
}

\author{
Jacqueline Solís-Céspedes \\ Tec Digital \\ Instituto Tecnológico de Costa Rica \\ Email: jacsolis@itcr.ac.cr
}

\author{
Julia Espinoza-Guzman \\ Tec Digital \\ Instituto Tecnológico de Costa Rica \\ Email: juliaespinoza@itcr.ac.cr
}

\begin{abstract}
Resumen-Learning styles have been used to explain students' differences in approaching their learning, but there are still deficiencies in interpreting the results of their application, and there are authors that indicate that there are no elements that support their credibility in achievement-based education. For this reason, we proposed a methodology to analyze the results obtained after the application of the test of learning styles. Our methodology is designed in four steps: identify the population and its characteristics, establish the time for the samples, determine the existing conglomerates in the population and to extract the characteristics of the constructed conglomerates. To evaluate the this aproach, the methodology was applied and validated by the Felder and Silverman's dichotomous test vs its diffuse version in an adult population in continuous formation and we found evidence that there are external aspects to those evaluated by the traditional learning styles instruments that could influence the changes of preferences over time.
\end{abstract}

Index Terms-Learning Styles, Felder Test, Learning Preferences, Mean Decrease GINI, Classification Process

\section{INTRODUCCIÓN}

Los estilos de aprendizaje se refieren "al modo en que aprende una persona -el tipo de procesamiento cognitivo que alguien usa durante el aprendizaje (tal como el estilo de verbalizar versus el de visualizar)" [1]. Los mismos autores indican, además, que lo importante en este caso se refiere a las diferentes habilidades cognitivas que implica cada estilo. Además, de acuerdo con lo que indican [2] quienes citan a [3] "el estudio de los estilos de aprendizaje constituye un vínculo entre dos ramas de la psicología, conocidas como cognición y personalidad; por lo que la forma en la que se razona y percibe la información puede variar con el tiempo [4]".

Los estilos de aprendizaje han sido utilizados para explicar las diferencias que tiene los estudiantes al enfocar su aprendizaje y incluso autores como [5] señalan que utilizar "una única estrategia para enseñar a todos los estudiantes puede tener un impacto negativo en los resultados de aprendizaje de aquellos estudiantes cuyas maneras de aprender divergen de la norma". Consecuentemente, para el estudiante también es relevante conocer su propio estilo predominante para utilizar recursos y aplicar procesos de aprendizaje que mejor aprovechen esas características. Pero la importancia de conocer el estilo de aprendizaje va más allá porque "han sido reconocidos como un factor humano que afecta el rendimiento de aprendizaje de los estudiantes" [6].
Desde el punto de vista psicológico existen múltiples enfoques para establecer las preferencias de aprendizaje de los estudiantes y estos enfoques han evolucionado a través de los años. Algunos ejemplos de los modelos utilizados para esta clasificación son los siguientes:

- Modelo basado en experimentación [7]: Fue propuesto por Kolb y sugiere que existen dos ejes para clasificar a los estudiantes: activa-observación reflexiva y la conceptualización abstracta-experiencia concreta. Es a partir de estos dos enfoques que se establecen cuatro tipos de preferencias: focalizador, acomodador, asimilador y ramificador.

- Modelo de Dewey [8]: Sugiere que el aprendizaje es un proceso que integra las experiencias, acciones y sentimientos y que los estudiantes aprenden mejor si se les incluye un componente de experiencia dentro de su proceso de aprendizaje.

- Modelo de Piaget [7], [9] : Desarrolló un modelo que explica las formas en que se puede organizar la adquisición de conocimientos. En este modelo se sugiere que la inteligencia es un aspecto fundamental en las interacciones de la persona con su entorno de aprendizaje.

- Modelo de los Cuadrantes Cerebrales de Herrmann [10]: La propuesta ofrece una escala para medir la preferencia en el funcionamiento mental y permite relacionar esta medida con los modos preferidos de conocimiento.

- Modelo de Programación Neurolingüística de Bandler y Grinder [11]: este modelo también es llamado visualauditivo-kinestésico (VAK) y define que tenemos tres sistemas para representar mentalmente la información: el visual, el auditivo y el kinestésico. "La PNL ofrece una comprensión conceptual ... basada en la ciencia de la información y la programación por computadoras, pero enraizada aún más en la observación de la experiencia de la vida humana"[11], que busca enseñar cómo se puede aprender a cambiar las experiencia, y a obtener control sobre lo que sucede en el cerebro a traves del pensamiento y la palabra.

- Modelo de las Inteligencias Múltiples de Gardner [12]: Este modelo considera que todos tenemos las ocho inteligencias en mayor o menor medida (lógica, lingüística, espacial, musical,corporal, interpersonal, intrapersonal, 
emocional y naturalista) y al igual que con los estilos de aprendizaje no hay tipos puros, ya si los hubiera les resultaría imposible funcionar.

- Modelo de Felder y Silverman [13], [14]: Es un modelo basado en los canales de percepción de información y clasifica los estilos de aprendizaje a partir de cinco dimensiones de carácter dicotómico (activoreflexivo, visual-verbal, sensitivo-intuitivo, inductivodeductivo, secuencial-global).

Tenemos entonces que la aplicación de teorías asociadas a diferentes corrientes psicológicas ha permitido ampliar las explicaciones de algunos fenómenos educativos, aunque este tema debe ser tratado como un proceso interdisciplinario en constante evolución y desarrollo [8], [15]. Es en este contexto que por años se ha aplicado el test Felder-Silverman [13], el cuál busca identificar el estilo particular de un persona para ofrecer actividades y recursos didácticos apropiados al estilo particular del estudiante, incluso hay experiencias de implementación del test de Felder en un LMS [16].

\section{I-A. Trabajo relacionado}

Existen diversos estudios que buscan establecer la relación entre enseñanza y aprendizaje con el propósito de que el profesor determine estrategias y recursos didácticos específicos a cada estilo de aprendizaje de sus estudiantes.

En este sentido, se encuentran expertos quienes apoyan su utilidad y argumentan que conocer el estilo de aprendizaje particular de los estudiantes puede guiar al docente a planificar y realizar actividades educativas apropiadas a cada estilo, a crear objetos de aprendizaje, crear contenidos educativos, o personalizar los cursos en el LMS [17], [18] y en la definición de sistemas adaptativos para estudiantes [19]. También existen iniciativas para mejorar las clasificaciones como el modelo propuesto por [20] y juegos educativos basados en estilos de aprendizaje [5] y [6].

En cuanto a la teoría asociada a la aplicación de los distintos mecanismos para identificar y favorecer las preferencias de un individuo existen tanto quienes apoyan su aplicación como quienes se oponen a su utilización. En este sentido, es importante rescatar que los modelos de clasificación se encuentran en un proceso de evolución constante y podemos encontrar modelos basados en la forma de la construcción del conocimiento, personalidad, bilateralidad cerebral, interacción con otras personas, estrategias de aprendizaje, canales de percepción de la información, aprendizaje organizacional, basados en la experiencia, entre otros; de modo que existen múltiples enfoques para afrontar dicha tarea [14].

\section{I-B. Argumentos detractores de los estilos de aprendizaje}

Según [21], las teorías referentes a los estilos de aprendizaje poseen fuertes deficiencias en cuanto a "a falta de sólida teoría explicativa, la falta de investigación que apoya la teoría, la falta de fiabilidad y la validez de las construcciones, y el fracaso en vincular los estilos de aprendizaje -la instrucción basada en el logro".

Además, la existencia de múltiples enfoques para realizar la evaluación de los estilos de aprendizaje también representa un reto para estandarizar los protocolos de análisis de resultados e interpretación de los mismos.

Para cada uno de los modelos existentes se tienen argumentos que justifican o contradicen los aspectos esenciales de su planteamiento original [7], [8], [9], [11], [12], [14], [20]. Por ejemplo, entre los detractores del Test Felder-Silverman podemos mencionar a Brito et al. [22], los cuáles, de acuerdo con su estudio de fiablidad detectaron que "los datos apoyan la confiabilidad de las dimensiones activo-reflexivo y visual-verbal, pero las dimensiones sensitivo-intuitivo y secuencial-global requieren re-evaluación”. Por lo que recomienda realizar más estudios "con poblaciones mayores de estudiantes que validen el cuestionario, a través de estimar tanto su validez como su confiabilidad. De igual forma, se recomiendan investigaciones que determinen si existe alguna relación positiva entre los estilos de aprendizaje y diversas estrategias de enseñanza".

Para el desarrollo de la presente propuesta se utilizó como referencia el modelo de Felder y Silverman, en sus versiones dicotómica y difusa.

La propuesta que se presenta no constituye una solución al problema de confiabilidad y validez de los instrumentos para determinar los estilos de aprendizaje, sino, que establece un marco común para la interpretación de los resultados obtenidos.

\section{I-C. Metodología Propuesta}

A continuación se presenta una propuesta para evaluar los resultados de la aplicación de los test de estilos de aprendizaje que puede ser utilizado como instrumento para comparar muestras de un mismo test en distintos momentos en el tiempo, o bien, establece un marco común para interpretar los resultados arrojados por distintos intrumentos relacionados con los estilos de aprendizaje.

I-C1. Paso 1: Establecer las características relevantes de la población a muestrear: Cada población posee características específicas que pueden resultar relevantes. Por ejemplo, en una población de estudiantes universitarios en transición a una vida profesional, los cambios sociales inciden en el rendimiento del personal y las preferencias de los mismos. Sin embargo, en una población escolar pueden existir otros factores que marquen las pautas para modificar sus preferencias.

Del mismo modo que en los procesos de análisis para minería de datos, en esta etapa se propone una evaluación de los intereses particulares de la población que se va a analizar. Se busca establecer una serie de características deseables a monitorear en forma paralela con la medición de los estilos de aprendizaje, de modo que se puede establecer si existen correlaciones o no entre las variables.

Se sugiere que para esta etapa se cuente con apoyo psicopedagógico, de modo que se puedan identificar los factores de estrés y detonantes de cambios específicos para las poblaciones de interés. 


\section{I-C2. Paso 2: Identificar los momentos de la muestra:}

Este paso consiste en establecer los momentos en los que será tomada la muestra para analizar los estilos de aprendizaje y contempla si será comparada con otra muestra en un momento en el futuro o no. Su valor viene intrínseco cuando se deben evaluar aspectos adicionales a la medición brindada por el instrumento de estilos de aprendizaje y permite una valoración de las modificaciones a través del tiempo.

\section{I-C3. Paso 3: Determinar los k-grupos existentes:} Esta fase consiste en determinar los $\mathrm{K}$ grupos existentes de acuerdo a las características de una población. Para eso existen múltiples algoritmos bien conocidos para este fin (como el uso de fórmulas de recurrencia para calcular el criterio de agregación, por ejemplo, el codo de Jambú [23] y la medida de distancia inter-clase). También se pueden utilizar librerías que ofrecen implementaciones plenamente funcionales que nos permiten establecer las características comunes de una población en particular.

\section{I-C4. Paso 4: Identificar los elementos determinantes para} la pertenencia a un grupo: Para determinar las variables que inciden en los resultados de un modelo predictivo se pueden aplicar diversas herramientas. Una técnica comúnmente utilizada consiste en medir la ganancia de información que aporta cada variable y analizar los centros de los clusters formados. Para efectos de nuestra prueba de propuesta, se va a trabajar utilizando como marco de referencia

\section{EVALUACIÓN DE LA PROPUESTA}

Para la evaluación de la propuesta se establecieron dos marcos de referencia: el primero, donde sólo se contempla la evaluación de los estilos de aprendizaje y una segunda, donde se incluyen aspectos que se consideró pueden afectar la percepción del entorno por parte del individuo como lo son los ejes de formación y los cambios sociales más relevantes que conyevan la transición de estudiantes a profesionales. A continuación se muestran los resultados generales encontrados al analizar los resultados de la aplicación del test de Felder en su modalidad dicotómica y difusa siguiendo la metodología de evaluación propuesta.

\section{II-A. Análisis dicotómico}

II-A1. Características de la población: La población evaluada consiste en un equipo de profesionales que se desempeñan en el área de e-learning. Sus áreas de expertise abarcan desde programadores hasta diseñadores industriales y se han mantenido en formación constante desde sus inicios en el ambiente laboral. Los individuos encuestados en este segmento poseen más de 3 años de experiencia laboral y forma parte de la planilla de la unidad, o bien, cambiaron recientemente sus labores hacia la docencia dentro de la misma universidad.
II-A2. Momento: Para población a evaluar se tomaron dos muestras, una en el 2014 y una segunda muestra en el 2017.

La muestra del 2014 estaba compuesta por la aplicación del test de felder dicotómico a diez personas de la unidad, sin embargo, para efectos de la evaluación fueron conservados únicamente seis registros a los que se les pudo dar continuidad en el 2017.

II-A3. k-grupos existentes: $\mathrm{Al}$ comparar los resultados de las muestras tomadas en el año 2014 contra las tomadas en el 2017 podemos apreciar cambios significativos con respecto a la conformación de los grupos.

En la figura 1 podemos apreciar la existencia de tres grupos de individuos conformados a partir de los valores disponibles en la muestra del año 2014. Estos grupos son un reflejo de las similitudes entre los estilos de aprendizaje registradas para los individuos encuestados.
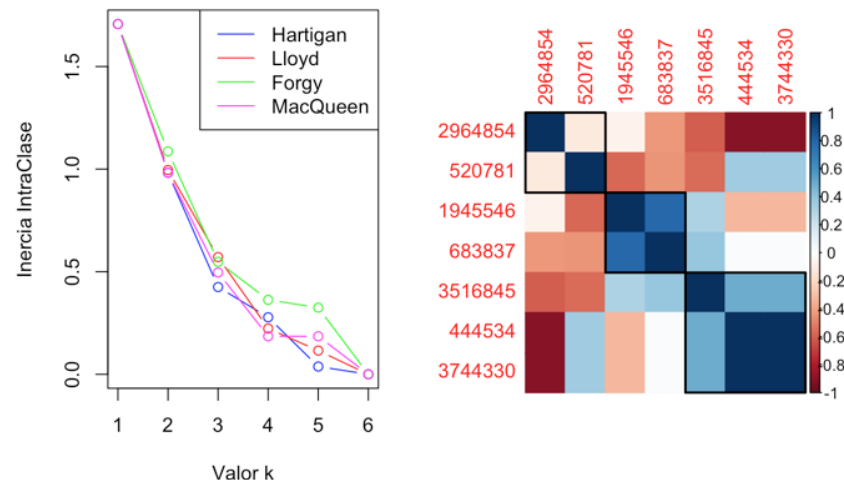

Figura 1. Mejor K para una población muestreada en el 2014 y la distribución de esos k-grupos sobre las correlaciones de la población

Por otro lado, en la figura 2 correspondiente al año 2017 podemos apreciar que se dieron cambios significativos en la distribución de los individuos dentro de los grupos. En este caso podemos apreciar que la población se redistribuyó en dos grupos bien delimitados, donde el grupo uno está muy marcado por correlaciones fuertes y positivas entre sus integrantes, y el segundo grupo donde la población presenta correlaciones débiles y positivas entre sus individuos.

Ambas mediciones contienen a la misma población, sin embargo, en el transcurso de la toma de la primera muestra con respecto a la segunda algún elemento indujo el cambio en las preferencias de la población, por lo que se necesita más información que la aportada por el test de Felder para establecer cuáles fueron los factores que motivaron el cambio.

II-A4. Identificar los elementos determinantes para la pertenencia a un grupo: Al establecer una evaluación basada solamente en la aplicación de los test de Felder dicotómicos se pierde la trazabilidad de los elementos sociales y psicológicos que pudieron incidir en los cambios de preferencias de la población muestreada.

Por este motivo, en la evaluación realizada en el 2017, se solicitó a los encuestados indicar los cambios sociales por los 
Mejor k usando Jambu

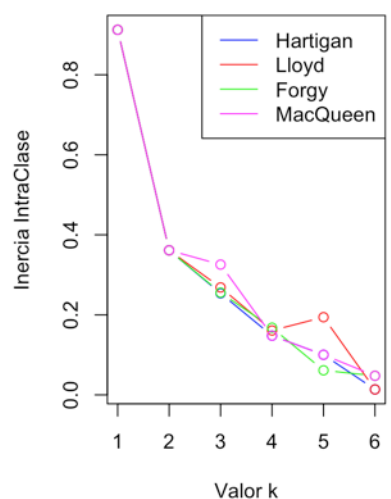

Figura 2. Mejor K para una población muestreada en el 2017 y la distribución de esos k-grupos sobre las correlaciones de la población

que había pasado en los últimos tres años, con el fin de agregar nuevas dimensiones al proceso de evaluación y contemplar un modelo que integre elementos de impacto psicológico para el individuo.

Los elementos consultados a los encuestados fueron:

- Rango de edad al que pertenece: Segmentado en bloques de 3 años para establecer los intervalos.

- Cambios significativos experimentados: Cambios familiares, lugar de trabajo o residencia, salud, entre otros.

- Área de trabajo: La población realiza tareas de producción de material audiovisual, programación, docencia, labores administrativas (gestión), entre otras.

- Eje de formación predominante: Dadas las características de la población, sus ejes de formación se han centrado en labores de gestión administrativa y de tecnologías de información, producción científica (investigadores), formación técnica y de idiomas.

- Cantidad de años transcurridos desde sus últimos estudios formales

- Grado Académico en el 2014 y Grado académico actual: Codificados desde 0 hasta 6 según el grado académico, donde 0 representa a los estudiantes universitarios en proceso de adquirir su título de ingeniería y 6 a los individuos con al menos un doctorado. La codificación completa se encuentra en el cuadro I e incluye todos los grados académicos presentes en la actualidad dentro del curriculum de los individuos encuestados.

\begin{tabular}{|c|c|}
\hline Grado académico & Identificador \\
\hline Estudiante universitario (sin título de grado) & 0 \\
Técnico & 1 \\
Diplomado & 2 \\
Bachillerato universitario & 3 \\
Licenciatura & 4 \\
Maestría & 5 \\
Doctorado & 6 \\
\hline
\end{tabular}

Cuadro I

CODIFICACIÓN DE GRADOS ACADÉMICOS
Estos elementos fueron estandarizados y se descartaron los elementos duplicados con la finalidad de eliminar ruido dentro el análisis de las relaciones entre las variables. De este modo, la muestra de datos pasó a contener elementos referentes a los estilos de aprendizaje recolectados en el 2014 y sus correspondientes cambios trazados mediante la encuesta hasta el 2017; y así, tras el preprocesamiento, se extrajeron 95 registros distintos con elementos atómicos por columna.
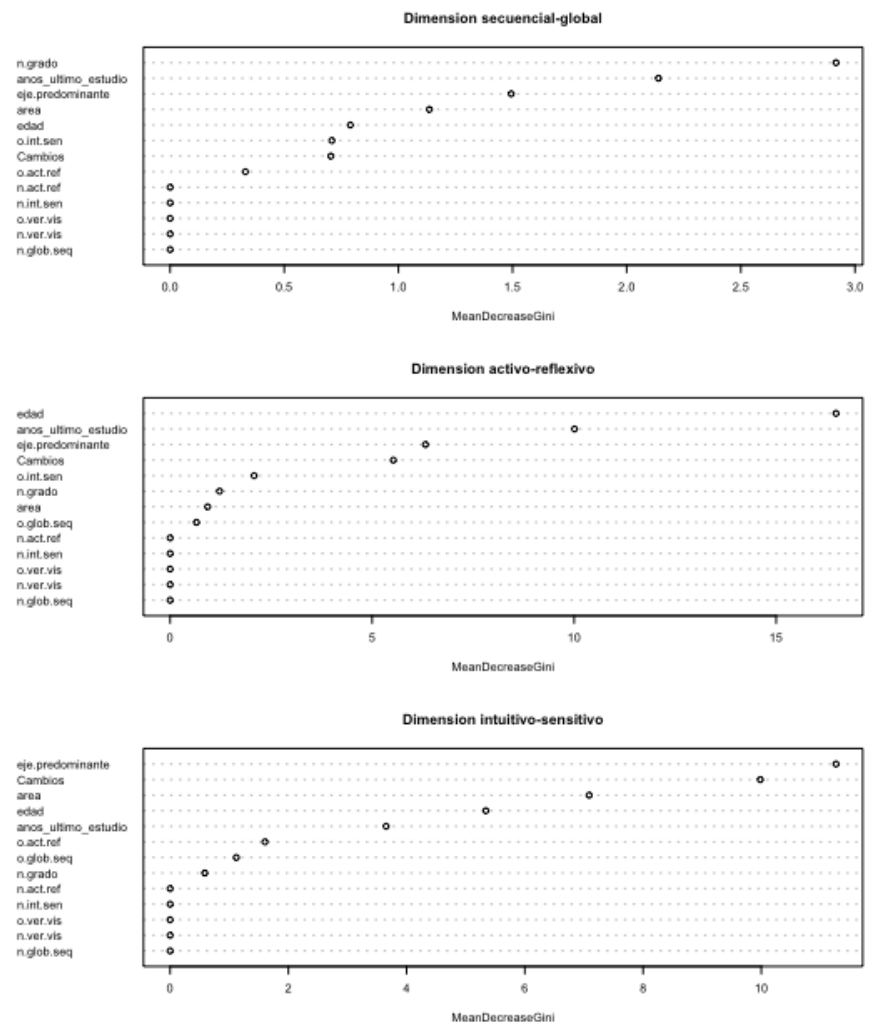

Figura 3. Importancia de las variables para las dimensiones activo-reflexiva, global-secuencial y sensitivo-intuitivo en el 2014

Al incorporar esta serie de elementos al modelo y ajustar los parámetros necesarios para un análisis de componentes jerárquico, se logró obtener una observación con una representatividad aproximadamente del $82 \%$ de los datos donde, se determinó que existen 4 conglomerados significativos que se pueden construir a partir de la totalidad de las variables involucradas en el análisis.

Para evaluar la importancia de cada variable se utilizó la medidad de ganancia de información mediante el índice Gini, aplicado a través del paquete de bosque aleatorio disponible en R. Un hallazgo derivado de este análisis fue que el eje de formación académico aparece dentro de las tres variables más importantes en la evaluación de las tres dimensiones medibles que fueron contempladas. Otras dos variables que aparecieron fueron la edad y la cantidad de años que han transcurrido desde sus últimos estudios. 

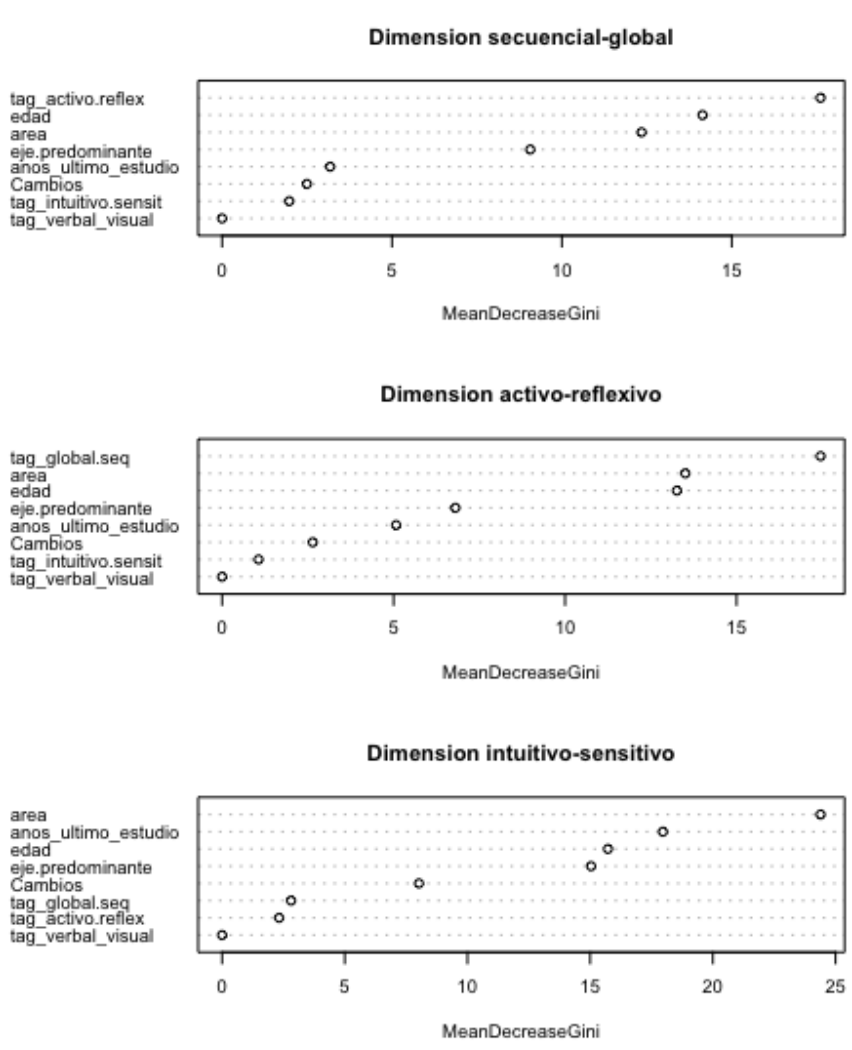

Figura 4. Importancia de las variables para las dimensiones activo-reflexivo, global-secuencial y sensitivo-intuitivo para muestra del año 2017

Para esta exploración fue excluida la dimensión verbal-visual, ya que la muestra de datos sólo contenía exponentes de tipo visual; razón por la cuál no se cuenta con un eje para comparar las diferencias entre sus exponentes.

\section{II-B. Ampliación de la muestra para Análisis dicotómico}

Tras la prueba inicial de concepto, se procedió a ampliar la muestra con la población que actualmente labora en la unidad de e-learning. Esta nueva muestra consiste en 27 individuos e incluye a los asistentes (estudiantes universitarios en proceso de adquirir sus títulos de grado).

Al realizar la ampliación de la muestra, el análisis jerárquico de componentes principales sugiere al existencia de tres conglomerados; a diferencia del modelo generado para la muestra del 2014, donde existían cuatro conglomerados, además, la variabilidad de los datos representados por las dos primeras componentes también disminuyó a un $58 \%$.

Además, el análisis de la importancia de las variables por dimensión parece existir una relación entre las dimensiones secuencial-global y la activo-reflexiva.

Entre otros aspectos relevantes, nuevamente las variables de edad, area y eje predominante de formación aparecen dentro de las variables con mayor relevancia como se puede apreciar en la figura 4 y la precisión de las predicciones realizadas sobre esta muestra supera el $96 \%$ en todos los casos como podemos apreciar en el cuadro II.

\begin{tabular}{|c|c|c|c|}
\hline Medida & activo-reflexivo & secuencial-global & intuitivo-sensivo \\
\hline Error de clase & $0 \%-13.9 \%$ & $0 \%-0 \%$ & $4.5 \%-2.6 \%$ \\
OOB & $2.97 \%$ & $0 \%$ & $3.47 \%$ \\
\hline
\end{tabular}

Cuadro II

MEDIDAS DE ERROR DE LA CLASIFICACIÓN

\section{II-C. Análisis del Test de Felder Difuso}

El instrumento aplicado corresponde a la variante del test de Felder y Silvenman propuesto por [20]. En esta variante, se asume la pertenencia difusa a las diferentes dimensiones por parte de los encuestados y provee etiquetas para la pertenencia a clases difusas más un valor normalizado asociado al valor de pertenencia a cada clase.

II-C1. Población y Momento: El momento de la muestra corresponde a una encuesta aplicada en el 2017 a la misma población utilizada para el análisis realizado en la sección II-B.

II-C2. k-grupos existentes: Para la prueba de concepto sobre un conjunto de datos de difuso no se incluyeron los elementos de cambios dado que no se cuenta con dos muestras en el tiempo para la misma población.

En este caso se contemplaron los indicadores de pertenencia para ambos extremos de las dimensiones de la especificación del test de Felder Difuso.
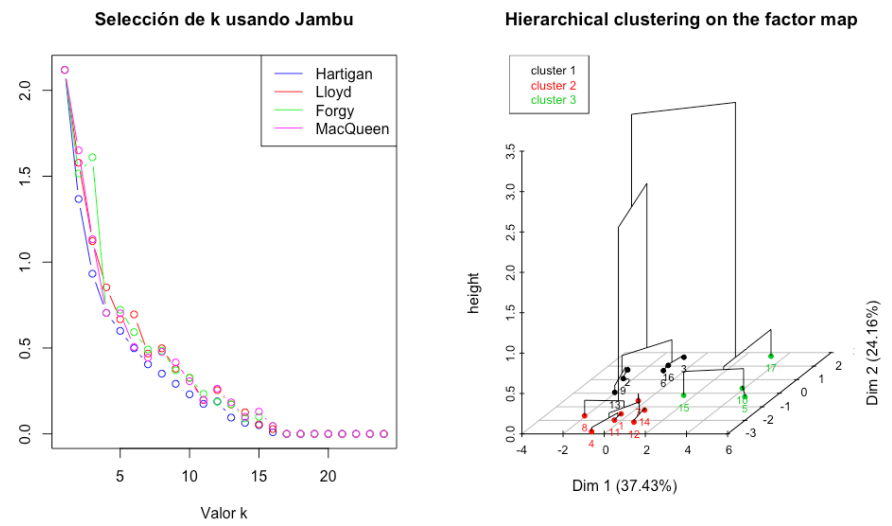

Figura 5. Selección de k para grupos en Felder difuso

En la figura 5 podemos apreciar que se identificaron tres conglomerados significativos dadas las características de la población. Aquí no tenemos valores inexistentes para alguno de los extremos, sin embargo, sí tenemos una muestra desbalanceada con las clases de pertenencia de un individuo para cada dimensión. Por ejemplo, del total de la muestra, todos los individuos pertenecen a la categoría activo moderado y reflexivo moderado, mientras que en el resto de clases pertenecen de forma débil a ambos extremos de la dimensión evaluada.

II-C3. Identificar los elementos determinantes para la pertenencia a un grupo: Al establecer los conglomerados 
para el conjunto de datos se encontraron tres grupos con correlaciones entre distintas dimensiones. El primero presenta una correlación débil y positiva entre la pertenencia a la clase activa y la visual. El segundo grupo presenta correlaciones débiles y positivas entre las dimensiones global, sensitiva e intuitiva $y$ un tercer grupo que posee correlaciones muy débiles y positivas entre las dimensiones reflexiva y secuencial.

La población monitoreada también muestra una correlación fuerte y negativa entre su pertenencia a la clase visual con respecto a la clase verbal. En un principio este es el comportamiento normal esperado para un test de Felder dicotómico, sin embargo, en el caso del test de Felder difuso aplicado esta es la única relación que cumple esta condición. Todas las demás correlaciones significativas presentan un comportamiento menos extremo.

Resulta interesante notar que la pertenencia a clases difusas

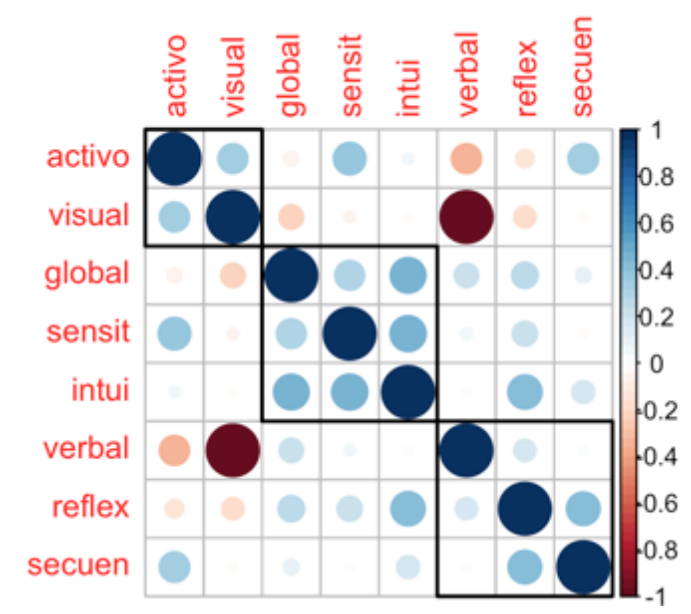

Figura 6. Correlaciones al aplicar Felder difuso en la población muestreada

correspondientes a los pares de dimensiones descritos por Felder no cumplen con el comportamiento de correlaciones fuertes y negativas esperado, de modo que podríamos intuir que la mayoría de los individuos encuestados poseen características cercanas a la media de pertenencia. Incluso se pueden observar pares de dimensiones (por ejemplo, la correlación entre la pertenencia a la clase sensitiva y la intuitiva) donde a pesar de tratarse de extremos dicotómicos opuestos en el test de Felder tradicional, presentan correlaciones de tipo fuerte y positivo en la muestra evaluada mediante el instrumento difuso.

Para este conjunto de datos no se contó con información adicional que permitiera la interpretación de la influencia de los ejes de formación o la edad con respecto a los valores incidentes para cada dimensión.

\section{CONCLUSIONES Y TRABAJOS FUTUROS}

En concordancia con lo publicado por los detractores del uso de los estilos de aprendizaje, la evaluación de dos muestras de datos de la misma población tomando en consideración sólo los resultados de un proceso de clasificación de estilos de aprendizaje no aporta información significativa que permita establecer los elementos causales de los cambios que se puedan apreciar. Para realizar una evaluación relevante de una población se necesita información adicional que no puede ser provista por sólo las etiquetas de pertenencia a clases (en el caso de los datos evaluados, las clases dicotómicas), ya que los elementos que fomentan los cambios en los estilos de aprendizaje parecen ser externos a dicha medición y con apoyo de expertos en psicología o pedagogía se podrían monitorear y aplicar en contextos fuera de las aulas.

Al incorporar variables para monitorear los cambios del entorno social en que se encuentran las personas al análisis de los estilos de aprendizaje, encontramos que elementos como la edad, el área de trabajo y eje predominante de formación académica recibido representan elementos relevantes en el proceso de modificación de las conductas y preferencias de aprendizaje.

Además, a diferencia de las dificultades para extraer resultados mediante el análisis dicotómico, el test de Felder Difuso permite evaluar todas las dimensiones representadas en el modelo (con inconvenientes menores asociados al volumen de la muestra); ya que existen valores de pertenencia para todas las dimensiones involucradas.

La estimación de los conglomerados a partir de los estilos de aprendizaje no siempre coincide con las dimensiones establecidas como parte del modelo de referencia. Se pueden dar situaciones donde las representaciones de las dimensiones desbalancean la construcción del modelo y esto está sujeto a las características de la población analizada.

La estandarización de los mecanismos para evaluar los estilos de aprendizaje aún es un área de estudio abierta y requiere de apoyo por parte de los tecnólogos para facilitar las herramientas que permitan una mejor comprensión de los datos existentes y de sus posibles aplicaciones en la vida real.

Como trabajo futuro se desea mejorar la estandarización del proceso de evaluación de los resultados de los distintos instrumentos de estilos de aprendizaje. De momento, los esfuerzos se han concentrado en la medición a partir de modelos basados en el Test de Felder, sin embargo, se espera mejorar el instrumento para que sea escalable a otros tipos de modelos. Además, se espera aplicar este modelo de análisis para estudiantes de distintas carreras de modo que se pueda establecer la influencia que tienen los ejes de formación definidos en los planes de estudio en sus preferencias de aprendizaje, formación y a futuro.

Las implicaciones de un modelo que permita estandarizar la evaluación de los cambios en los estilos de aprendizaje podrían modificar la forma en que se establecen los planes de estudio en los distintos niveles académicos y permitiría mejorar la comprensión sobre los mecanismos de adquisión de conocimiento en la población. Además, un mecanismos que permita el intercambio de percepciones de distintos modelos de evaluación de los estilos de aprendizaje permitiría el enriquecimiento de las perspectivas de evaluación para segmentos de la población que tienen dificultades, o caen dentro de los 
parámetros atípicos de medición por parte de los modelos de estilos de aprendizaje.

\section{REFERENCIAS}

[1] R. E. Mayer and L. J. Massa, "Three facets of visual and verbal learners: Cognitive ability, cognitive style, and learning preference." Journal of educational psychology, vol. 95, no. 4, p. 833, 2003.

[2] J. Solís-Céspedes, "Agente recomendador híbrido de objetos de aprendizaje," 2014.

[3] R. J. Sternberg and E. L. Grigorenko, "Are cognitive styles still in style?" American psychologist, vol. 52, no. 7, p. 700, 1997.

[4] H. A. Witkin and D. R. Goodenough, "Cognitive styles: essence and origins. field dependence and field independence." Psychological issues, no. 51, pp. 1-141, 1980.

[5] M. Soflano, T. M. Connolly, and T. Hainey, "Learning styles in adaptive games-based learning," in International Joint Conference SOCO'13CISIS'13-ICEUTE'13. Springer, 2014, pp. 709-717.

[6] G.-J. Hwang, H.-Y. Sung, C.-M. Hung, I. Huang, and C.-C. Tsai, "Development of a personalized educational computer game based on students' learning styles," Educational Technology Research and Development, vol. 60, no. 4, pp. 623-638, 2012.

[7] D. A. Kolb, Experiential learning: Experience as the source of learning and development. FT press, 2014.

[8] C. C. Liu, I. Ju et al., "Evolution of constructivism," Contemporary issues in education research, vol. 3, no. 4, p. 63, 2010.

[9] J. Piaget and F. F. Buey, Psicología y pedagogía. Ariel Barcelona 1969.

[10] A. Silva Sprock, J. C. Ponce Gallegos, and M. D. Villalpando Calderón, "Modelo para la creación y uso de objetos de aprendizaje, basado en la valoración de técnicas instruccionales," Conferencias LACLO, vol. 3 , no. 1, 2012.

[11] R. Bandler and J. Grinder, Use su cabeza para variar. Cuatro Vientos, 1988.

[12] H. Gardner, "Inteligencias múltiples," 1995.

[13] R. M. Felder et al., "Learning and teaching styles in engineering education."

[14] M. A. P. Ospina, L. I. D. Salazar, and J. S. C. Meneses, "Modelos de estilos de aprendizaje: una actualización para su revisión y análisis," Revista Colombiana de educación, no. 64, pp. 79-105, 2013.

[15] F. D. B. Arceo and G. H. Rojas, "Estrategias docentes para un aprendizaje significativo una interpretación constructivista," 1999.

[16] O. C. Santos, J. G. Boticario, E. Raffene, and R. Pastor, "Why using dotlrn? uned use cases," Este libro se distribuye bajo licencia Creative Commons Reconocimiento CompartirIgual 2.5 Espa, p. 195.

[17] G. G. Vargas, "Uso de las tic como recurso pedagogico para el aprendizaje de la cartografia tematica aplicada al estudio de las geociencias: oportunidades y retos," Revista de Topografía AZIMUT, vol. 7, no. 1, pp. 60-66, 2017.

[18] D. L. Arreaga, L. De la Fuente Valentín, A. Pardo, and C. D. Kloos, "Adaptación de material educativo guiada por ims learning design: Experiencias con. lrn (adaptation of learning material guided by ims learning design: Experiences with. 1rn)," Revista Iberoamericana de Educación a Distancia, vol. 13, no. 2, p. 209, 2010.

[19] H. M. González, N. D. Duque, and D. A. Ovalle, "Modelo del estudiante para sistemas adaptativos de educación virtual," Avances en Sistemas e Informática, vol. 5, no. 1, 2008.

[20] A. Silva, R. M. Vicari, and J. C. Ponce, "Fuzzy learning styles questionnaire based in felder and silverman model," in Learning Objects and Technology (LACLO), Latin American Conference on. IEEE, 2016, pp. $1-7$.

[21] D. An and M. Carr, "Learning styles theory fails to explain learning and achievement: Recommendations for alternative approaches," Personality and Individual Differences, 2017.

[22] M. D. Brito-Orta and R. Espinosa-Tanguma, "Evaluación de la fiabilidad del cuestionario sobre estilos de aprendizaje de felder y soloman en estudiantes de medicina," Investigación en educación médica, vol. 4, no. 13 , pp. 28-35, 2015.

[23] M. Jambu and M.-O. Lebeaux, Classification automatique pour l'analyse des données. Dunod Paris, 1978, vol. 1. 